\title{
Incorporation of Unnatural Amino Acid into Antibody Fragment for Creating a Stable Antibody-Drug Conjugate
}

\author{
Andri Wardiana1,2*, Martina L Jones ${ }^{2,3}$, Stephen M Mahler ${ }^{2,3}$, and Christopher B Howard ${ }^{2,3}$
}

1. Research Centre for Biotechnology, Indonesian Institute of Sciences (LIPI). Jl. Raya Bogor Km 46, 16911, West Java, Indonesia

2. Australian Institute for Bioengineering and Nanotechnology (AIBN), The University of Queensland, 4072 QLD, Australia

3. ARC Training Centre for Biopharmaceutical Innovation, Corner College and Cooper Rds (Bldg 75), The University of Queensland, Brisbane, QLD 4072, Australia

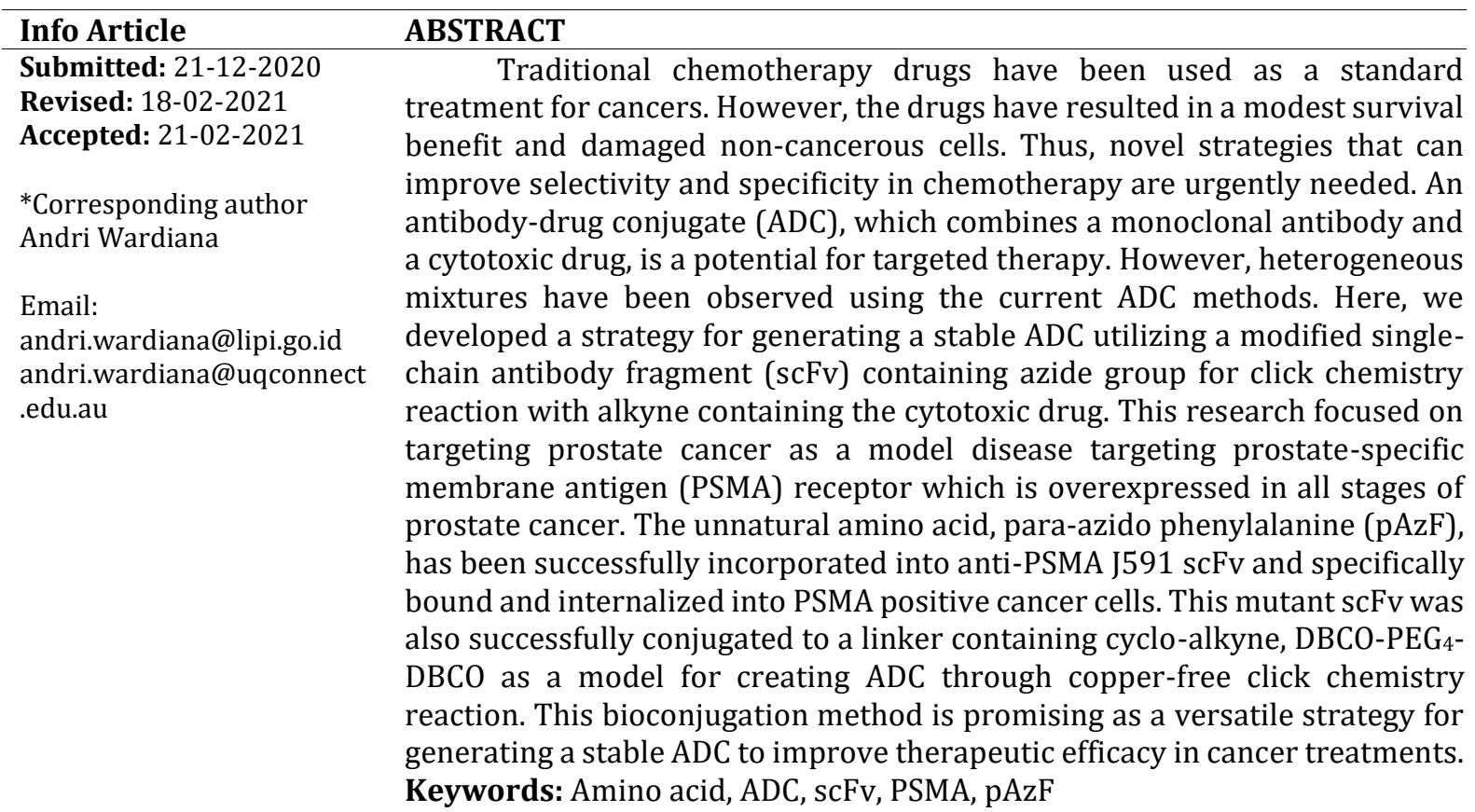

\section{INTRODUCTION}

Cancer is a non-communicable disease that is estimated to rank as the leading cause of death worldwide. In most cases, lifestyle-related factors including tobacco consumption and obesity remain the major contributors to cancers. In addition, environmental factors (e.g. air and water pollution), bacterial and viral infections, as well as family history can also induce cancers in humans (Brown et al., 2018; Parsa, 2012). It has been reported that there were 19.3 million incidences of cancer worldwide in 2020 and 10 million deaths (GLOBOCAN, 2020). Furthermore, the global cancer burden is projected to reach 21 million cases and 13 million deaths in 2030 with breast, prostate, and lung cancer remain as the major cases (Rahib et al., 2014; Zarocostas, 2010).
Cancer development is associated with the accumulation of genetic mutations and epigenetic changes resulting in the loss of control in cellular growth of cancer cells. These cancer cells can invade normal tissues and organs, and spread throughout the body (Hanahan and Weinberg, 2011; Sharma et al., 2009). There are a hundred different types of cancer, which can give distinct responses with various treatments, including surgery, radiotherapy, immunotherapy, and chemotherapy. The deployment of monoclonal antibodies (mAbs) has revolutionized cancer therapy, with the first clinical trial in 1992. According to a report in 2017, there are around 30 monoclonal antibodies that have been approved and are marketed for the treatment of various types of cancers (Falzone et al., 2018). In 2018, 33 novel antibody therapeutics for cancer treatments 
were in late-stage clinical trials, with around $20 \%$ of the total were projected to enter regulatory review in 2019 and 2020 (Kaplon and Reichert, 2019). The mAbs can selectively bind to the target receptors that are overexpressed on the tumor cell surface and subsequently kill the tumor cells through several mechanisms including complement-dependent cytotoxicity, antibodydependent cell-mediated cytotoxicity, and several altered signal transductions (Scott et al., 2012). However, most mAbs therapies show insufficient therapeutic activity against specific tumor receptors (Scott et al., 2012). By conjugation of a cytotoxic drug to the mAb, which is known as an antibody-drug conjugate (ADC), the efficacy and potency of $m A b$ can be improved. ADC is designed for targeted, cytotoxic drug delivery into tumor cells without harming the healthy tissues. ADCs preferentially deliver cytotoxic drugs through tumor-associated antigens to achieve improved drug efficacy and safety. Several ADCs have been approved for cancer treatment, such as brentuximab vedotin (Adcetris/SGN-35; an antiCD30 antibody conjugated with the microtubuledisrupting agent monomethyl auristatin E) for Hodgkin's lymphoma. Several other ADCs are currently in late-stage clinical trials. In general, synthesis methods for ADCs involve modification of lysine or cysteine residues which leads to a distribution of zero to eight toxins per antibody at various sites. However, heterogeneous mixtures of conjugates have been observed through these reactions, especially when modifying large proteins such as antibodies (Dan et al., 2018). Therefore, the ability to synthesize homogeneous ADCs is important to optimize the efficacy, safety, and pharmacokinetics of the drug conjugates.

Since the last decades, recombinant methods have been used to generate bioconjugates via the incorporation of unnatural amino acids (UAA) into proteins as chemical handles for bio-orthogonal conjugation reactions. In principle, the UAA can be incorporated at any desired position in any protein. Thus, using genetically encoded UAAs for bioorthogonal protein conjugation we can generate chemically defined, homogeneous proteins. Furthermore, the incorporation of UAA conserves the structure of the wild-type protein since it does not require any particular sequence context to specify the UAA site, and site-specific modified proteins are expressed recombinantly in high yields in bacteria, yeast, or mammalian cells (Liu and Schultz, 2010).
In this study, we developed a novel bioconjugation method that can be utilized for ADC synthesis. We focused on prostate cancer as a model disease by targeting prostate-specific membrane antigen (PSMA) receptors using an antiPSMA J591 antibody. PSMA has been widely investigated as an excellent target for therapy with monoclonal antibody approaches since this transmembrane protein is overexpressed in all stages of prostate cancer (Ross et al., 2003; Troyer, Beckett, and Wright, 1995). Herein, we described the expression of anti-PSMA J591 antibody singlechain variable fragments (scFvs) in Escherichia coli, with incorporated UAA para-azido phenylalanine (pAzF) to facilitate the generation of a stable conjugate of anti-PSMA-pAzF with imaging dye or linker, as a proof of concept for ADC platform technology. The $\mathrm{pAzF}$ was genetically encoded into $\mathrm{scFv}$ by an engineered tRNA/aaRS pair derived from M. jannaschii. This engineered pair can incorporate $\mathrm{pAzF}$ in response to the amber codon TAG situated upstream at the N-terminus of the $\mathrm{scFv}$ gene coding sequences. Subsequently, the antibody fragment scFv-imaging dye/linker conjugates were generated by the formation of a strong triazole linkage following strain-promoted, copper-free click chemistry reaction between the azide functional group in the pAzF incorporated scFvs and cycloalkyne functional group in the dibenzocyclooctyl (DBCO) containing fluorescent dyes or linker. In this study, the characterization and verification of pAzF incorporation into J591 $\mathrm{scFv}$ were investigated, as well as the successful biorthogonal reactions with DBCO containing dye/linker.

\section{MATERIALS AND METHODS Cell lines}

Human prostate cancer cell lines, PC3 (ATCC CRL-1435) which are PSMA-negative cells, and PC3-PIP (PC3 transfected with human PSMA) which are PSMA-positive cells, were cultured in RPMI 1640 (Gibco) containing $10 \%$ fetal bovine serum (Gibco). All cell lines were incubated at $37^{\circ} \mathrm{C}$ in $5 \% \mathrm{CO}_{2}$.

\section{Linker}

DBCO-PEG4-DBCO (Jena Bioscience) linkerconjugate with $\mathrm{MW}$ around $0.9 \mathrm{kDa}$ was used as a proof-of-concept for click chemistry reaction between azide-modified J591 scFv and a large molecule containing DBCO group. 


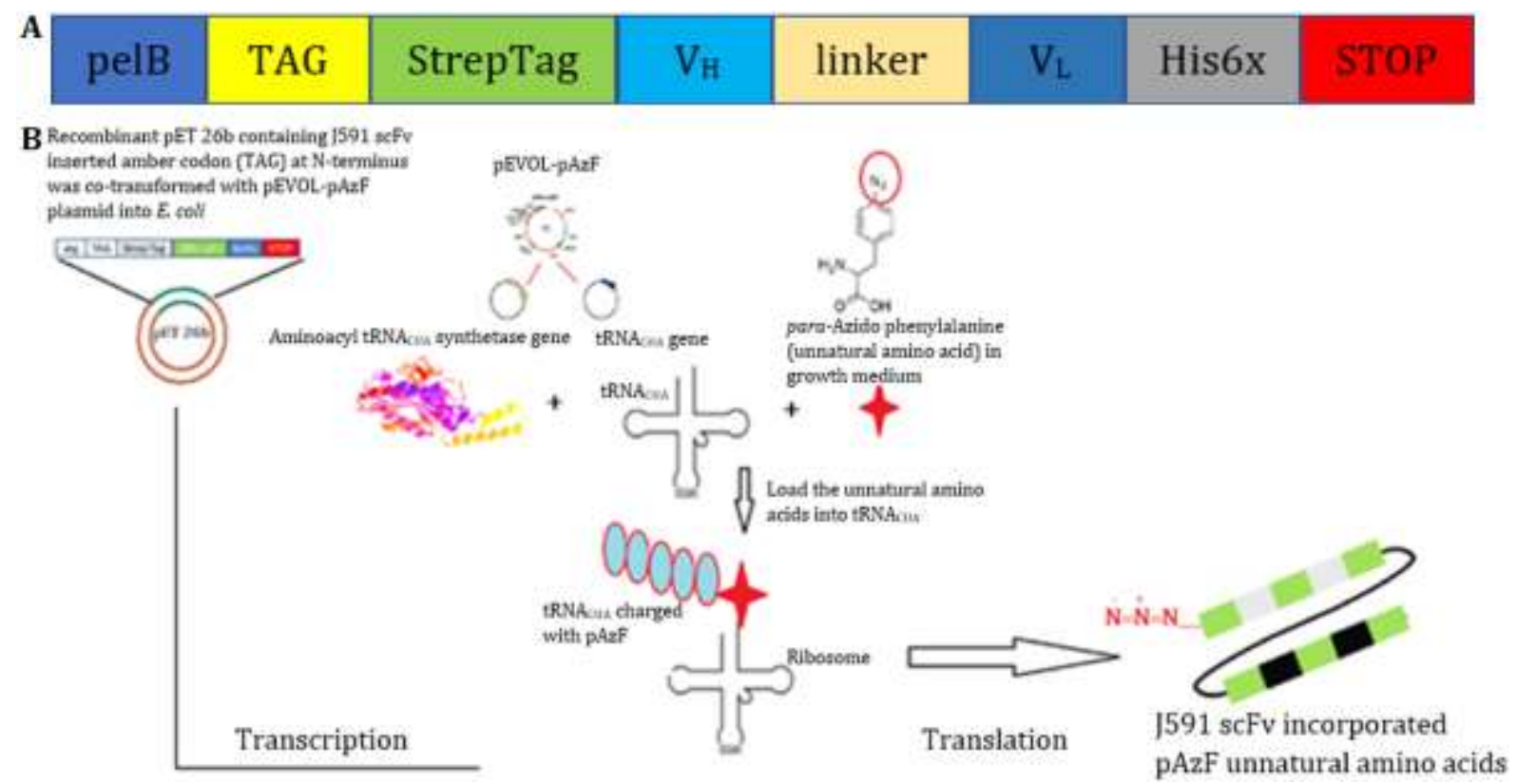

Figure 1. Design, cloning and expression of anti-PSMA. J591 scFv incorporated pAzF in periplasmic space of E. coli system. A) Plasmid design, pelB; promoter for periplasmic expression; TAG: amber stop codon for unnatural amino acid attachment site; StrepTag: strep tag coding sequence for purification purposes; VH: variable heavy chain sequence of J591 antibody; linker: flexible linker (G4S)3; VL: variable light chain of J591 antibody; His6: Histidine $6 \mathrm{X}$ tag for purification and detection purposes; Stop: stop codon. B) A schematic of unnatural amino acid (UAA) incorporation method in periplasmic $E$. coli expression system. Plasmid encoding J591 scFv were co-transformed with plasmid encoding the suppressor tRNA and amino acyl-tRNA synthetase (aaRS). The UUA pAzF were added into growth medium for subsequent tRNA acylation and its incorporation into J591 scFv.

\section{Cloning and protein expression of anti-PSMA J591 scFv-incorporated pAzF}

The anti-human PSMA J591 scFv sequence as mentioned above was synthesized and codonoptimized for $E$. coli expression by Geneart. Amber codon (TAG) was incorporated at the N-terminus by PCR followed by the strep tag coding sequence and a $6 \mathrm{x}$ His tag coding sequence incorporated at the C-terminus for purification and detection purposes. The amplified gene was cloned into pET26b for periplasmic protein expression to produce soluble proteins. Recombinant pET26b containing J591 ScFv with amber codon was cotransformed into BL21 (DE3)-competent cells with pEVOL-pAzF (Addgene, Schultz lab collection, kindly provided by Dr. Angus Johnson) encoding a tRNA synthetase/tRNA pair for the in vivo incorporation of p-azido-l-phenylalanine (pAzF) in response to the amber codon (Figure 1). Colonies were grown on LB medium with kanamycin $(30 \mu \mathrm{g} / \mathrm{mL})$ and chloramphenicol $(34 \mu \mathrm{g} / \mathrm{mL})$. A single colony was grown in LB liquid until the $\mathrm{OD}_{600}$ reached $0.5-0.8$, followed by the addition of $1 \mathrm{mM}$ of
IPTG (Astral Scientific), $0.05 \%$ of L-arabinose (Sigma ), and $3 \mathrm{mM}$ of pAzF (Iris Biotech, $\mathrm{GmbH}$ ), then was incubated at $20^{\circ} \mathrm{C}, 200 \mathrm{rpm}$ for $16-20 \mathrm{~h}$. Cultured cells were harvested by centrifugation at $4000 \mathrm{xg}$ for $10 \mathrm{~min}$. The soluble protein was extracted from the periplasm by resuspending cells thoroughly in $5 \mathrm{~mL}$ of ice-cold sucrose solution per $1 \mathrm{~g}$ cell pellet containing $30 \mathrm{mM}$ Tris- $\mathrm{HCl} \mathrm{pH} 8.0$, $20 \%(\mathrm{w} / \mathrm{v})$ sucrose, and $1 \mathrm{mM}$ EDTA. The sample was incubated on ice for $30 \mathrm{~min}$ and then centrifuged at $13.000 \mathrm{xg}, 4^{\circ} \mathrm{C}$ for $20 \mathrm{~min}$. The sucrose fraction was kept and stored on ice. After that, the cell pellets were dissolved in $5 \mathrm{~mL}$ of ice-cold $5 \mathrm{mM} \mathrm{MgSO}_{4}$ per $1 \mathrm{~g}$ cell pellets and $1 \mathrm{x}$ protease inhibitor cocktail (Roche), then lysozyme (Sigma) was added at a final concentration of $100 \mu \mathrm{g} / \mathrm{mL}$. The sample was incubated on ice for $10 \mathrm{~min}$ and then centrifuged at $13.000 \mathrm{xg}, 4^{\circ} \mathrm{C}$ for $20 \mathrm{~min}$. The supernatant was kept on ice and combined with sucrose fraction. The sample was then dialyzed against $1 \mathrm{X}$ D-PBS overnight at $4^{\circ} \mathrm{C}$, then purified by Strep-tag affinity chromatography. 


\section{Protein purification}

The protein was purified using StrepTrap ${ }^{\mathrm{TM}}$ HP 5mL column (GE Healthcare) connected to an AKTA purifier system, following provided manufacturer's methods. Briefly, the column was equilibrated with a binding buffer containing $1 \mathrm{X}$ PBS (20mM sodium phosphate, $280 \mathrm{mM} \mathrm{NaCl}, 6 \mathrm{mM}$ potassium chloride) $\mathrm{pH}$ 7.4. Then, the filtered sample was applied into the column, and the flowthrough solution was collected. The column was washed with binding buffer (wash fraction was collected), followed by an elution step using a binding buffer containing $2.5 \mathrm{mM}$ desthiobiotin, and the elution fractions were stored. The purified protein was also characterized using size exclusion chromatography (SEC) (Superdex 75 10/300, GE Healthcare), connected to the AKTA purifier system using $1 \mathrm{X}$ PBS as the binding and elution buffers.

\section{Flow cytometry analysis of J591-scFv-pAzF}

Cell monolayer of grown PC3 (PSMAnegative) and PC3-PIP (PSMA-positive) cells were removed with enzyme-free cell dissociation buffer (Gibco), centrifuged at $500 \mathrm{rpm}$ for $4 \mathrm{~min}$, and resuspended in fresh $10 \%$ FBS-PBS to give a concentration of $2 \times 10^{6}$ cells $/ \mathrm{mL}$. scFv at a concentration of $1 \times 10^{-6} \mathrm{M}$ were added to $100 \mu \mathrm{L}$ of cells $\left(2 \times 10^{6}\right.$ cell $\left./ \mathrm{mL}\right)$ and treated as described previously. FITC-labeled anti-His antibody (Miltenyi Biotec $\mathrm{GmbH}$ ) was used as the secondary antibody. Then cells were analyzed on the Accuri C6 Flow Cytometer using FL1 (533/30 nm) optical filter to detect the FITC-labeled secondary antibody. Data were evaluated using FCS Express 4 Flow software.

\section{Click chemistry reaction between J591 scFv- pAzF and Cy5-labeled DBCO}

The incorporation of pAzF into J591 scFv was evaluated by copper-free click chemistry reaction using Cy5-labeled-DBCO (Sigma-Aldrich). $10 \mu \mathrm{g}$ of purified protein was mixed with $1 \mu \mathrm{L}$ of Cy5-labeled-DBCO, then incubated at $4^{\circ} \mathrm{C}$ overnight. The reaction was evaluated by SDSPAGE and detected through Cy5 channel protein imaging (ChemiDoc Imaging system, Bio-Rad).

\section{Binding and internalization of Cy5-labeled DBCO conjugated with J591-scFv-pAzF into prostate cancer cells}

Target binding efficiency of the conjugates was performed using flow cytometry analysis. The conjugates were incubated in PC3 and PC3-PIP cells and treated with the protocol described above without the secondary antibody incubation step. The cells were then analyzed on CytoFlex Flow Cytometer Platform (Beckman Coulter) through APC channel $(690 / 50)$ to detect Cy5. Data were evaluated using FCS Express 4 Flow software.

For the internalization study, the Cy5labeled DBCO-conjugated J591-scFv-pAzF were incubated in the grown cells (PC3 and PC3-PIP cells) with around $80 \%$ confluence at $37^{\circ} \mathrm{C}, 7.5 \%$ $\mathrm{CO}_{2}$ for $4 \mathrm{~h}$. Following the incubation, cells were washed with $1 \mathrm{x}$ PBS three times, then fixed with $4 \%$ paraformaldehyde (PFA) solution $0.5 \mathrm{~mL}$ for $20 \mathrm{~min}$ at room temperature. The PFA was removed and the cells were washed with 1x PBS three times. Finally, the coverslips were mounted on slides that had been applied with Vectashield $\AA$ and DAPI (Cole-Parmer). Slides were observed and imaged under a confocal microscope (Leica TCS SP8) at the Australian National Fabrication Facility's Queensland Node (ANFF-Q).

\section{Click chemistry reaction of DBCO-PEG linker and J591 scFv-pAzF}

Twenty micro liters $(20 \mu \mathrm{L})$ of $10 \mu \mathrm{M}$ J591pAzF was mixed with $2 \mu \mathrm{L}$ of $10 \mathrm{mM}$ DPD, then incubated at $37^{\circ} \mathrm{C}$ overnight with shaking at $300 \mathrm{rpm}$. The unconjugated DPD was removed through the $0.5 \mathrm{~mL}$ Zeba spin desalting column with 7kDa MWCO (Thermosfisher), following provided manufacturer's protocol. The reaction was incubated again for another night under the same reaction conditions. Conjugated and unconjugated scFvs were then analyzed using SDS-PAGE.

\section{Verification of J591 scFv-pAzF-DPD conjugation by mass spectrometry}

Protein $(\sim 1 \mu \mathrm{g})$ was analyzed using an Orbitrap Elite (Thermo) mass spectrometer and Dionex Ultimate 3000 nano-LC system (Thermo). The sample was first desalted on a C4 PepMap300 pre-column (300 um x 5mm, 5um 300A) using buffer A (30 $\mu \mathrm{L} / \mathrm{min})$ for $5 \mathrm{~min}$ and separated on a C4 Acclaim PepMap 300 (75 $\mu \mathrm{m}$ x $150 \mathrm{~mm}$ ) at a flow rate of $300 \mathrm{~nL} / \mathrm{min}$. A gradient of $10-70 \%$ buffer $B$ over $10 \mathrm{~min}$, followed by $70-98 \%$ buffer $\mathrm{B}$ over $0.5 \mathrm{~min}$ was used, where buffer A was $0.1 \%$ formic acid in water and buffer B was 80\% acetonitrile $/ 0.1 \%$ formic acid. Eluted protein was directly analyzed on an Orbitrap Elite (Thermo) mass spectrometer interfaced with a NanoFlex source. The MS was operated in positive ion mode using the ion trap analyzer. Source parameters included an ion spray voltage of $2.4 \mathrm{kV}$, temperature at $275^{\circ} \mathrm{C}, \mathrm{SID}=30 \mathrm{~V}$, S-lens $=70 \mathrm{~V}$, 
summed micro scans $=3$, FT vacuum $=0.1 \mathrm{MS}$ analysis was performed across $800-2000 \mathrm{~m} / \mathrm{z}$. Data were deconvoluted using Thermo Protein Deconvolution ${ }^{\mathrm{T} M}$ software across mass 1100 $1800 \mathrm{~m} / \mathrm{z}$ with a minimum of $6-10$ adjacent charges, target mass of 30 or $60 \mathrm{kDa}$ as needed, and charge state range of 10-100. Deconvoluted data were reported as average masses.

\section{RESULTS AND DISCUSSION}

Modified ScFv production and bioactivity assay The incorporation of UAA into proteins and subsequent production in various cellular expression systems has proven to be a novel method for protein engineering. Through residuespecific incorporation, the engineering of both physical and chemical properties of proteins is possible, while incorporation at specific sites minimizes structural changes, thus conserving functionality. In this study, we used pAzF providing azide functionality, which was incorporated at the $\mathrm{N}$-terminus of J591 anti-PSMA scFv. The incorporation of azide-bearing UAA facilitates biorthogonal click chemistry reactions via azidealkyne cycloaddition. Since the TAG amber codon is a stop codon, the position of the TAG codon at the upstream of the gene encoding scFv ensures the generation of only homogeneous products, that is only anti-PSMA J591 scFv incorporated with pAzF. In addition, the incorporation of azide at the $\mathrm{N}$ terminal of $\mathrm{scFv}$ is favorable, as this position is exposed to the solvent and generally accessible for further chemical modifications (Rosen and Francis, 2017).

Incorporation of azide-containing unnatural amino acid, pAzF, into J591 scFv was carried out using a periplasmic E. coli expression system, by cotransforming the pEVOL-pAzf plasmid encoding the aminoacyl-tRNA synthetase/suppressor tRNA pair and an appropriate plasmid containing TAG amber codon at the $\mathrm{N}$-terminus, into competent cells BL21 (DE3). A positive clone was selected and expressed in LB medium with appropriate inducers and an optimized amount of pAzF. The cells were harvested, followed by periplasmic extraction using the combination of EDTA-lysozyme and cold osmotic shock. The overall yield of J591 scFv-pAzF in periplasmic $E$. coli was up to $5-6 \mathrm{mg} / \mathrm{L}$. Protein expression in a periplasmic E. coli production system obtains soluble proteins at a high yield, avoids protein aggregation, and suppresses the formation of dimers. In agreement with a previous study, soluble J591 scFv-pAzF expressed well in the periplasmic space of E. coli, with a low amount of dimers and the absence of aggregates. However, our results showed that the overall yield of soluble periplasmic J591 scFv-pAzF was about half of the yield of soluble periplasmic wild-type $\mathrm{scFv}$ previously reported by Frigerio and colleagues (Frigerio et al., 2013). This study showed that in general, expressing protein with UAA incorporation using amber codon has a limitation in terms of protein yield (Young and Schultz, 2010). The main reason for the low yield of UAAincorporated protein in comparison to the wild type is presumably due to an inefficient translation caused by an incompatibility of orthogonal synthetase and its suppressor tRNA pairs with host cell organisms (Smolskaya and Andreev, 2019). In addition, when standard periplasmic E. coli expression systems are utilized, our mutant scFv product is mostly expressed as an inclusion body in the cytoplasmic space (data not shown). The tendency for protein accumulation as insoluble protein is probably due to the lack of processing signal peptidase, resulting in the J591 scFv-pAzF failed to translocate into the periplasmic space of $E$. coli. However, this study suggests that the expression of J591 scFv-pAzF in the periplasmic expression system is still superior compared to that in the cytoplasmic expression systems (Baneyx and Mujacic, 2004). The disadvantages of expressing in the cytoplasmic space are the need for resolubilization from inclusion bodies and refolding of the protein, which is an inefficient process, and the presence protein aggregates and dimers. However, the most important reason for the unsuitability of cytoplasmic expression is the instability of the azide group in the presence of a reducing agent, such as dithiothreitol (DTT), which an essential agent in inclusion body solubilization and recombinant protein is refolding. DTT, used for the reduction of disulfide bonds in this mutant $\mathrm{scFv}$, can reduce the azide in $\mathrm{pAzF}$ to a corresponding amine group, resulting in non-functional azide and prohibiting chemical conjugation reactions (Cartwright et al., 1976; Staros et al., 1978). Therefore, we found that the efficient production of azide-bearing UAA-incorporated J591 scFv was in the periplasmic E. coli expression system.

The J591 scFv pAzF extracts were purified using Strep-tagged protein purification, resulting in a protein preparation with high purity (Figure $2 \mathrm{~A}$ ). The purified $\mathrm{scFv}$ was characterized by size exclusion chromatography, confirming that the purified $\mathrm{scFv}$ preparation was predominantly monomeric protein with a small percentage of dimers and protein aggregates (Figure $2 \mathrm{~B}$ ). 

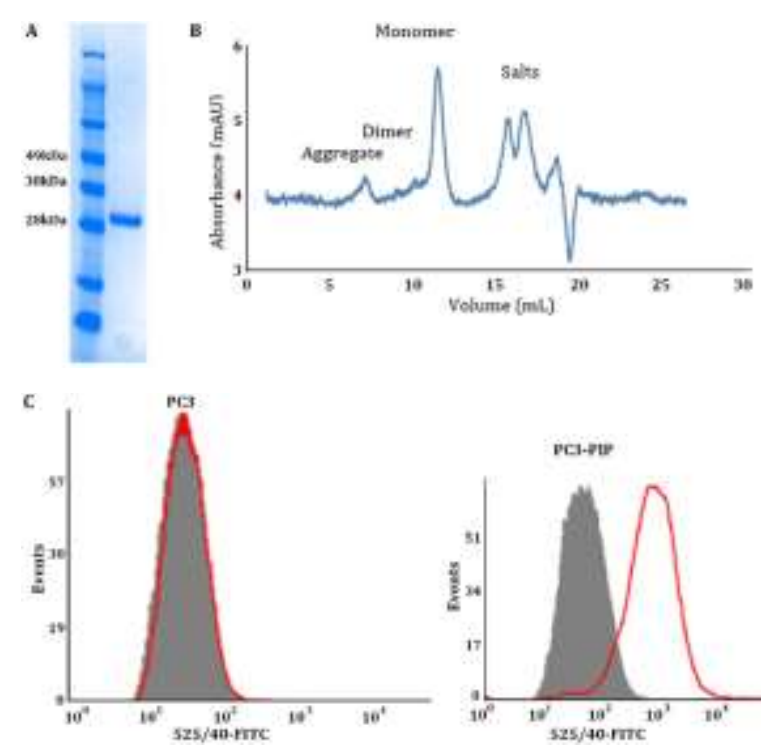

Figure. 2. Purification and affinity binding assay of J591 scFv-pAzF. A) SDS PAGE of purified J591 scFvpAzF showing $\sim 28 \mathrm{kDa}$ in MW. B) Size exclusion chromatography shows that the scFv expressed in periplasmic space contains mainly monomeric protein with low protein aggregates and protein dimers. C) Flow cytometry analyses indicated that J591 scFv-pAzF can specifically bind into PSMA positive human prostate cancer cell line (PC3-PIP), but not into negative cell line (PC3). Histogram, grey: cells with PBS (control); red: cells with J591 scFv pAzF.

To evaluate targeting efficiency into membraneexpressed PSMA, the binding specificity of J591scFv was verified using flow cytometry, utilizing PSMA-expressing prostate cancer cells (PC3-PIP) and PSMA-negative prostate cancer cells (PC3), as the negative control. The purified mutant $\mathrm{scFv}$ showed binding activity to PSMA-expressing prostate cancer cells PC3-PIP and showed no binding to PSMA-negative, PC3 prostate cancer cells as shown in Figure 2C. This binding study proved that the incorporation of azide-bearing unnatural amino acids at the $\mathrm{N}$-terminus preserves the specific binding activity of the J591 anti-PSMA scFv.

\section{Verification of azide-modified unnatural amino acid incorporation by click chemistry reaction toward DBCO containing imaging-dye}

To confirm the site-specific incorporation of UAA pAzF into anti-PSMA J591 scFv, the click chemistry reaction of dibenzocyclo-octyne (DBCO) derivative toward mutant $\mathrm{ScFv}$ was examined. A biorthogonal reaction was performed to confirm the incorporation of azide UAA into J591 scFv using copper-free cycloaddition by employing cycloalkyne from $\mathrm{DBCO}$, which is highly reactive towards the azide group (Figure $3 \mathrm{~A}$ ). This catalystfree click reaction avoids the use of the cytotoxic $\mathrm{Cu}(\mathrm{I})$ catalyst, which has been widely used in alkyne-azide cycloaddition. DBCO containing cyanine dye (Cy5) was used for the copper-free click chemistry reaction and mixed with either wild-type J591 scFv or J591 scFv-pAzF. Detection under the Cy5 channel confirmed that only mutant $\mathrm{scFv}$ containing azide group had been clicked with the DBCO group (Figure 3 B). DBCO-labeled Cyanine 5 dye was mixed with scFv; wild-type J591 $\mathrm{scFv}$ was used as a negative control. Coomassie blue staining clearly showed the bands of both mutant and wild-type scFvs. However, fluorescent imaging of mutant scFv revealed a clear band, while the wild-type scFv did not show any fluorescent labeling (Figure 3 B). Therefore, the results confirmed that the azide-containing UAA has been successfully incorporated into J591 scFv.

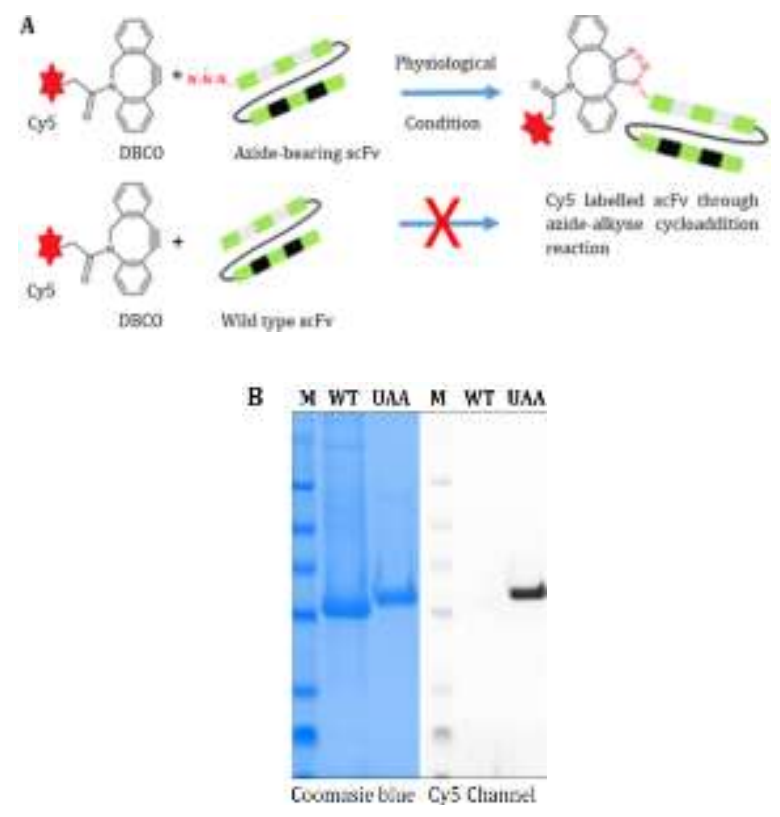

Figure 3. Evaluation of UAA pAzF attachment into a single-chain fragment variable (scFv) of J591 antibody and its binding analysis. A) Copper-free click chemistry reaction of Cy5 labelled DBCO with J591 scFv-pAzF. B) Comparison between J591 scFv wild type and J591 scFv-pAzF in coomassie blue and chemiluminescence Cy5 channel; $\mathrm{M}$ : protein marker; WT: J591 scFv wild type; UAA: J591 scFvpAzF. 

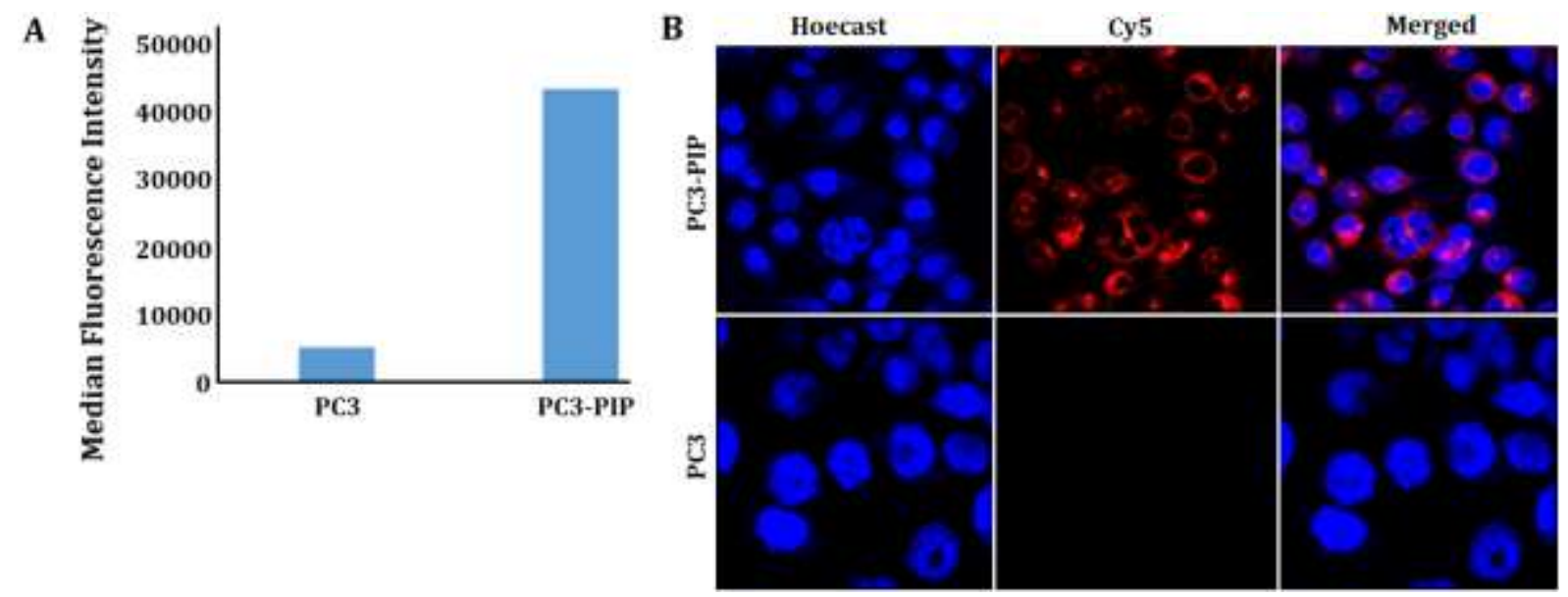

Figure 4. In vitro binding specificity and internalization studies of azide-modified J591-scFv conjugated into Cy5-labelled DBCO. A) Flow cytometry assays showed that the scFv dye conjugates specifically binds into PSMA positive prostate cancer cells (PC3-PIP) and not into PSMA negative prostate cancer cells (PC3). B) Immunofluorescence analyses also showed the specificity of scFv dye conjugate in PSMA expressing prostate cancer cells (PC3-PIP).

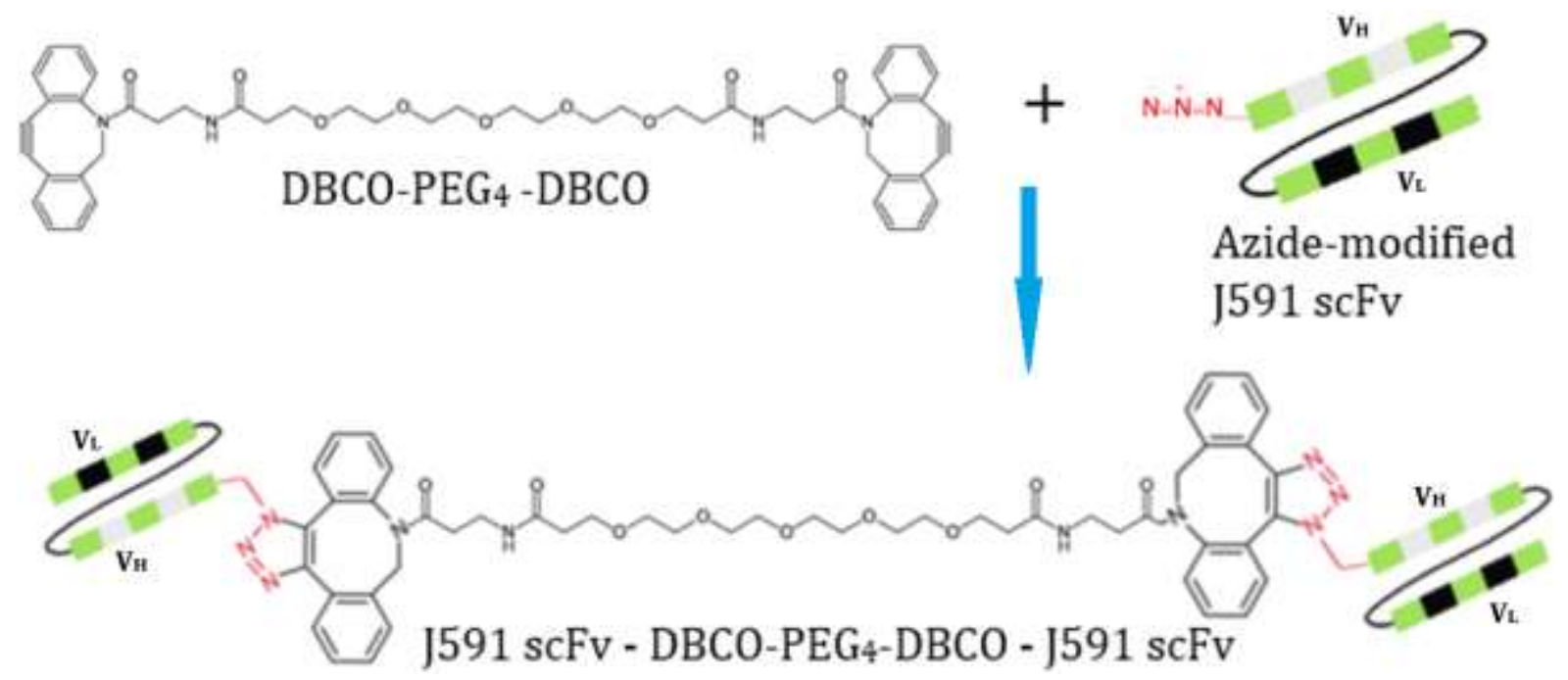

Figure 5. Copper-free click chemistry reaction of J591 scFv-pAzF and DBCO-PEG linker under physiological condition.

In vitro internalization study of scFv-dye conjugate uptake into prostate cancer cells

In vitro binding studies were performed to evaluate the J591 scFv-pAzF conjugated with Cy5labeled DBCO using flow cytometry and confocal microscopy. The internalization of J591 scFv-pAzFDBCO-Cy5 conjugate evaluated by immunofluorescence read-out flow cytometry analysis through Cy5 channel (Figure 4 A) indicated that the conjugates specifically bound to
PSMA-expressing prostate cancer cells. In addition, immunofluorescence staining imaged using a confocal microscope showed that the conjugate was fully internalized into PSMA-positive PC3-PIP cells, while no binding and internalization into PSMA-negative PC3 cells was evident (Figure 4 B). Results showed that the scFv was labeled with Cy5 dye, confirming the successful conjugation and specific binding and internalization into PSMApositive prostate cancer cells, PC3-PIP. 

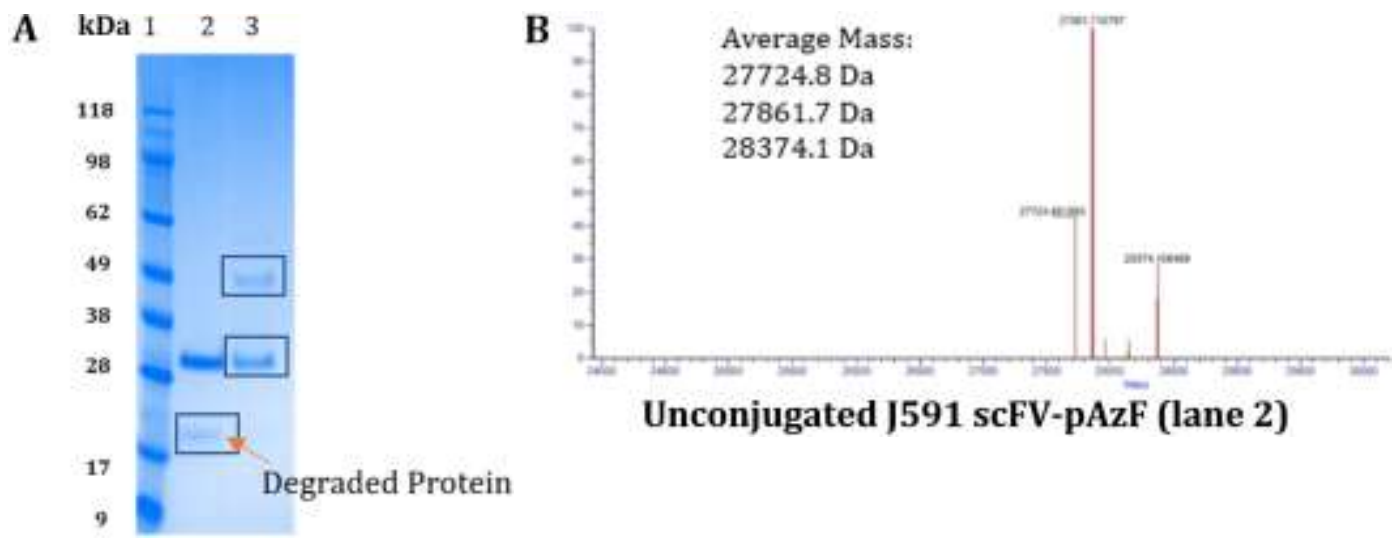

\section{Unconjugated J591 scFV-pAzF (lane 2)}
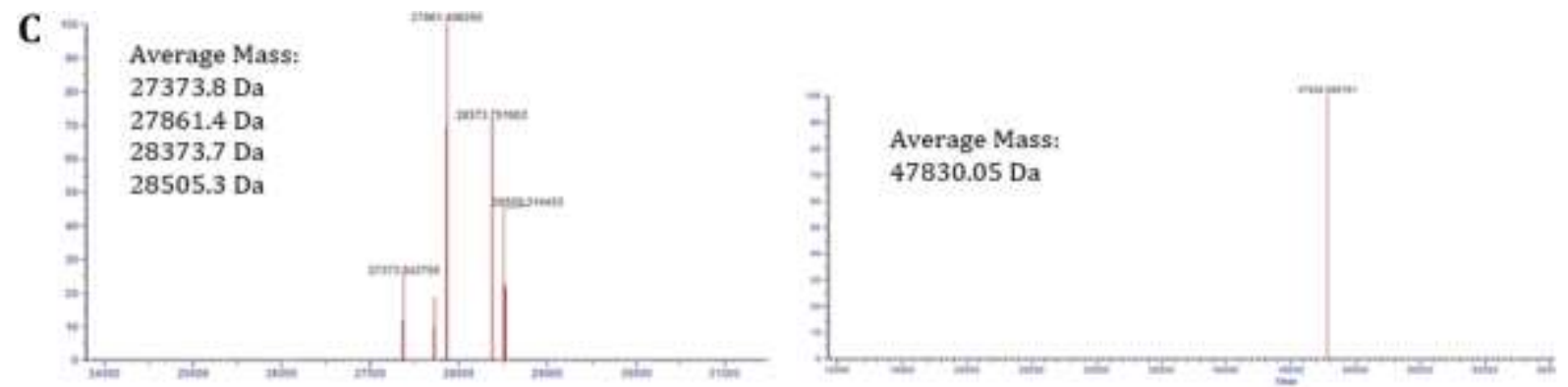

Conjugated J591 scFv-pAzF (lane 3)

Figure 6. Verification of conjugation of DBCO-PEG4-DBCO with J591 scFv-pAzF. A) SDS PAGE show the unconjugated and conjugated scFvs. Lane 1: Marker; lane 2: unconjugated scFv; lane 3: conjugated scFv shows several bands containing excess scFv (unconjugated) and scFv-linker conjugates. B) Deconvoluted masses for unconjugated scFv as reported by Protein Deconvolution software. C) Deconvoluted masses for conjugated of scFv-DPD as reported by Protein Deconvolution software, measuring two MW species including excess scFv (unconjugated), and a higher MW species of $\sim 47 \mathrm{kDa}$.

\section{Conjugation of azide-modified J591 scFv and DBCO PEG linker}

To verify whether J591 scFv-pAzF can be used to attach other molecules, a DBCO PEG linker with MW around $1 \mathrm{kDa}$ was chosen as a testbed for larger molecules in the copper-free click chemistry reaction. The linker has two DBCO molecules in the opposite arms which can be coupled into azidebearing scFv (Figure 5). To evaluate the conjugation, SDS-PAGE analysis was performed. Results showed that more than one band appeared on the gel for the conjugated sample as can be seen in Lane 3 in Figure 6A. In addition, the less intense band of degraded protein of unconjugated $\mathrm{scFv}$ was also detected, as can be seen on the gel with lower MW at $\sim 19 \mathrm{kDa}$ (Lane 2). Further analysis was then performed using intact mass spectrometry to verify the MW of each band. In the unconjugated scFv, the intact mass was shown to be around $27-28 \mathrm{kDa}$.
However, lower MW species for degraded protein were not detected (Figure 6B). In the conjugated scFv, the intact mass results showed the same average mass with unconjugated $s c F v$, i.e. around 27-28kDa, and one larger species with an average mass of $47 \mathrm{kDa}$ (Figure 6C). These results confirmed there were excess unconjugated scFvs in the mixture of products, and some scFvs were successfully conjugated with the $1 \mathrm{kDa}$ DBCO PEG linker on both sides. However, it is presumed that one side of DBCO reacted with $27 \mathrm{kDa} \mathrm{scFv}$ and another side of $\mathrm{DBCO}$ reacted with lower MW of degraded $\mathrm{scFv}$ which was around $19 \mathrm{kDa}$ based on the SDS-PAGE gel result.

While this conjugation reaction requires further scrutiny, the azide group incorporated at the N-terminus of scFv showed the accessibility for interaction with larger molecules, such as cytotoxic drugs containing DBCO. This site-specific 
conjugation through click chemistry reaction has the potential to minimize a heterogenous ADC. The stability and homogeneity of ADC are crucial to improving therapeutic effects.

\section{CONCLUSION}

There are myriad of covalent conjugation methods that have been widely researched to obtain a stable conjugate for successful ADC. For conjugation, a suitable functional group has to be incorporated into the $\mathrm{mAb}$ or cytotoxic drug. In this research, we have successfully incorporated a UAA in vivo in E. coli expression system to functionalize the antibody fragment anti-PSMA J591 scFv with the azide group at the $\mathrm{N}$-terminus, providing biorthogonality and a higher degree of specificity. Additionally, we have improved the production efficiency of this modified scFv by expressing the protein in the periplasmic space to obtain highly monomeric protein yields.

In this study, we have successfully conjugated the azide-modified J591 scFv with DBCO containing imaging dye or linker through copper-free cycloaddition click chemistry reaction, which proved high accessibility of azide moiety in this mutant scFv for the conjugation reaction. In future studies, ADC reaction will be performed by bioconjugation of anti-PSMA J591 scFv-pAzF into a cytotoxic drug. For example, an antimitotic agent, monomethyl auristatin E (MMAE), will be used as the drug. A previous study showed that MMAE could inhibit the proliferation of human prostate cancer (Cunningham et al., 2016). MMAE-DBCO containing linker will be conjugated to anti-PSMA J591 scFv-pAzF using copper-free click chemistry reaction to yield ADCs for targeted prostate cancer therapy.

\section{ACKNOWLEDGMENT}

Elements of this research utilized the facilities, and the scientific and technical assistance of the National Biologics Facility (NBF), University of Queensland. NBF is supported by Therapeutic Innovation Australia (TIA). TIA is supported by the Australian Government through the National Collaborative Research Infrastructure Strategy (NCRIS) program. This work was also performed in part at the Queensland nodes of the Australian National Fabrication Facility (ANFF), a company established under the National Collaborative Research Infrastructure Strategy to provide nano and micro-fabrication facilities for Australian researchers.

Andri Wardiana received a scholarship from the
Indonesia Endowment Fund for Education (LPDP) for the doctoral study at the University of Queensland, Australia.

\section{AUTHOR CONTRIBUTIONS}

AW is the main contributor and prepared the manuscript with contributions from all authors. AW and $\mathbf{C B H}$ designed the experiments. AW, $\mathbf{C B H}$, SMM, and MLJ conceived the project.

\section{REFERENCES}

Baneyx, F., and Mujacic, M. (2004). Recombinant protein folding and misfolding in Escherichia coli. Nat Biotechnol, 22(11), 1399-1408. doi:10.1038/nbt1029

Brown, K. F., Rumgay, H., Dunlop, C., Ryan, M., Quartly, F., Cox, A., ... Parkin, D. M. (2018). The fraction of cancer attributable to modifiable risk factors in England, Wales, Scotland, Northern Ireland, and the United Kingdom in 2015. British Journal of Cancer, 118(8), 1130-1141. doi:10.1038/s41416018-0029-6

Cartwright, I. L., Hutchinson, D. W., and Armstrong, V. W. (1976). The reaction between thiols and 8-azidoadenosine derivatives. Nucleic Acids Res, 3(9), 2331-2339. doi:10.1093/nar/3.9.2331

Cunningham, D., Parajuli, K. R., Zhang, C., Wang, G., Mei, J., Zhang, Q., . . Y You, Z. (2016). Monomethyl Auristatin E Phosphate Inhibits Human Prostate Cancer Growth. The Prostate, 76(15), 1420-1430. doi:10.1002/pros.23226

Dan, N., Setua, S., Kashyap, V. K., Khan, S., Jaggi, M., Yallapu, M. M., and Chauhan, S. C. (2018). Antibody-Drug Conjugates for Cancer Therapy: Chemistry to Clinical Implications. Pharmaceuticals, 11(2), 32.

Falzone, L., Salomone, S., and Libra, M. (2018). Evolution of Cancer Pharmacological Treatments at the Turn of the Third Millennium. Frontiers in Pharmacology, 9(1300). doi:10.3389/fphar.2018.01300

Frigerio, B., Fracasso, G., Luison, E., Cingarlini, S., Mortarino, M., Coliva, A., ... Figini, M. (2013). A single-chain fragment against prostate specific membrane antigen as a tool to build theranostic reagents for prostate cancer. European Journal of Cancer, 49(9), 22232232. doi:https://doi.org/10.1016/j.ejca.2013.01. $\underline{024}$ 
GLOBOCAN 2020: New Global Cancer Data. (2020). Retrieved from https://www.uicc.org/news/globocan2020-new-global-cancer-data\#

Hanahan, D., and Weinberg, Robert A. (2011). Hallmarks of Cancer: The Next Generation. Cell, 144(5), 646-674. doi:https://doi.org/10.1016/j.cell.2011.02. $\underline{013}$

Kaplon, H., and Reichert, J. M. (2019). Antibodies to watch in 2019. mAbs, 11(2), 219-238. doi:10.1080/19420862.2018.1556465

Liu, C. C., and Schultz, P. G. (2010). Adding New Chemistries to the Genetic Code. Annual Review of Biochemistry, 79(1), 413-444. doi:10.1146/annurev.biochem.052308.105 824

Parsa, N. (2012). Environmental factors inducing human cancers. Iranian journal of public health, 41(11), 1-9.

Rahib, L., Smith, B. D., Aizenberg, R., Rosenzweig, A. B., Fleshman, J. M., and Matrisian, L. M. (2014). Projecting Cancer Incidence and Deaths to 2030: The Unexpected Burden of Thyroid, Liver, and Pancreas Cancers in the United States. Cancer Research, 74(11), 2913-2921. doi:10.1158/0008-5472.can14-0155

Rosen, C. B., and Francis, M. B. (2017). Targeting the $\mathrm{N}$ terminus for site-selective protein modification. Nature Chemical Biology, 13(7), 697-705. doi:10.1038/nchembio.2416

Ross, J. S., Sheehan, C. E., Fisher, H. A. G., Kaufman, R. P., Kaur, P., Gray, K., ... Kallakury, B. V. S. (2003). Correlation of Primary Tumor Prostate-Specific Membrane Antigen Expression with Disease Recurrence in Prostate Cancer. Clinical Cancer Research, 9(17), 6357-6362.
Scott, A. M., Wolchok, J. D., and Old, L. J. (2012). Antibody therapy of cancer. Nat Rev Cancer, 12(4), 278-287. doi:10.1038/nrc3236

Sharma, S., Kelly, T. K., and Jones, P. A. (2009). Epigenetics in cancer. Carcinogenesis, 31(1), 27-36. doi:10.1093/carcin/bgp220

Siegel, R. L., Miller, K. D., and Jemal, A. (2020). Cancer statistics, 2020. CA Cancer J Clin, 70(1), 7-30. doi:10.3322/caac. 21590

Smolskaya, S., and Andreev, Y. A. (2019). SiteSpecific Incorporation of Unnatural Amino Acids into Escherichia coli Recombinant Protein: Methodology Development and Recent Achievement. Biomolecules, 9(7), 255.

Staros, J. V., Bayley, H., Standring, D. N., and Knowles, J. R. (1978). Reduction of aryl azides by thiols: Implications for the use of photoaffinity reagents. Biochemical and Biophysical Research Communications, 80(3), 568-572.

doi:https://doi.org/10.1016/0006291X(78)91606-6

Troyer, J. K., Beckett, M. L., and Wright, G. L., Jr. (1995). Detection and characterization of the prostate-specific membrane antigen (PSMA) in tissue extracts and body fluids. Int J Cancer, 62(5), 552-558. doi:10.1002/ijc.2910620511

Young, T. S., and Schultz, P. G. (2010). Beyond the Canonical 20 Amino Acids: Expanding the Genetic Lexicon*. Journal of Biological Chemistry, 285(15), 11039-11044. doi:https://doi.org/10.1074/jbc.R109.0913 $\underline{06}$

Zarocostas, J. (2010). Global cancer cases and deaths are set to rise by $70 \%$ in next 20 years. $\quad B M J, \quad 340, \quad$ c3041. doi:10.1136/bmj.c3041 